\title{
Enzyme Activity to Augment the Characterization of tethered Bilayer Membranes:
}

\section{Supplementary material}

Gintaras Valincius, Duncan J. McGillivray, Wilma Febo-Ayala, David J. Vanderah, John J.

Kasianowicz, and Mathias Lösche

(A) Chemical synthesis

Figure S1: A short scheme of the synthesis of WC14

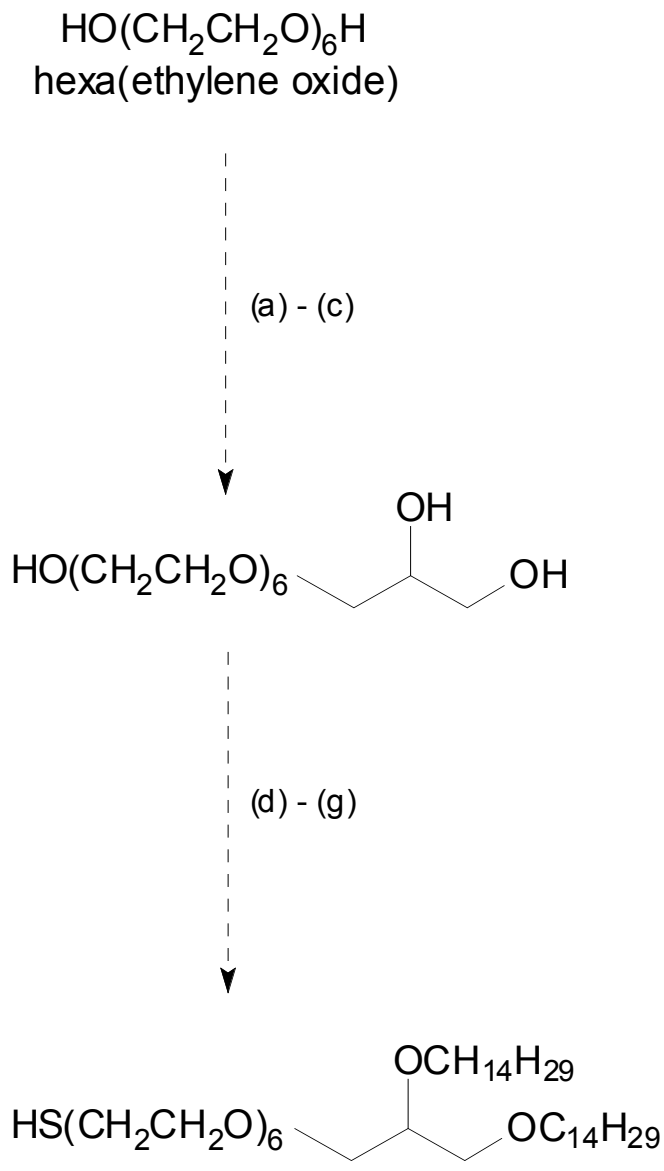

1

(a) $\mathrm{NaH}, \mathrm{BrCH}_{2} \mathrm{CH}=\mathrm{CH}_{2} / \mathrm{THF}$; (b) 3,4-dihydro-2H-pyran $/ \mathrm{CHCl}_{3}$; (c) $\mathrm{OsO}_{4-}$ 4-methylmorpholine-N-oxide/acetone- $\mathrm{H}_{2} \mathrm{O}$-t-butanol; (d) $\mathrm{C}_{14} \mathrm{H}_{29} \mathrm{OSO}_{2} \mathrm{CH}_{3} /$ $\mathrm{C}_{6} \mathrm{H}_{6} ;(\mathrm{e}) \mathrm{CH}_{3} \mathrm{CO}_{2} \mathrm{H} / \mathrm{THF} / \mathrm{H}_{2} \mathrm{O}(4 / 2 / 1)$; (f) $\left(\mathrm{CF}_{3} \mathrm{CO}_{2}\right)_{2} \mathrm{O} / \mathrm{THF}$ then $\mathrm{Li} \mathrm{Br} / \mathrm{THF}-$ HMPA; (g) 3 eq. $\mathrm{CH}_{3} \mathrm{COS}^{-} \mathrm{Na}^{+} / \mathrm{CH}_{3} \mathrm{OH}$. 
(B) Electrochemical Impedance Spectroscopy: Equivalent circuit models and model parameters for the data shown in Figure 2a and $b$

\section{(a) Precursor SAM}

Figure S2: Equivalent circuit

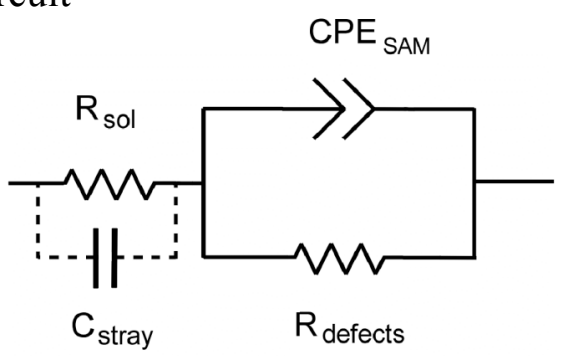

Table S1: Model parameters*

\begin{tabular}{lcl}
\hline Parameter & Value & \multicolumn{1}{c}{ Units } \\
\hline$R_{\text {sol }}$ & $30.9(1)$ & $\Omega \cdot \mathrm{cm}^{2}$ \\
$C_{\text {stray }}$ & $0.0105(6)$ & $\mu \mathrm{F} \cdot \mathrm{cm}^{-2}$ \\
$C P E_{\mathrm{SAM}}$ & $8.70(5)$ & $\mu \mathrm{F} \cdot \mathrm{cm}^{-2} \cdot \mathrm{s}^{(\alpha-1)}$ \\
$\alpha_{\mathrm{SAM}}$ & $0.987(1)$ & \\
$R_{\text {defects }}$ & $0.44(8)$ & $\mathrm{M} \Omega \cdot \mathrm{cm}^{2}$ \\
\hline
\end{tabular}

*Shown is a representative set of fitting parameters obtained from a single EIS spectrum of a SAM. The data are normalized to the geometric surface area, $a$. The surface roughness factor is $\beta \sim 1.49$. 
b) Completed tBLMs:

Figure S3: Equivalent circuit

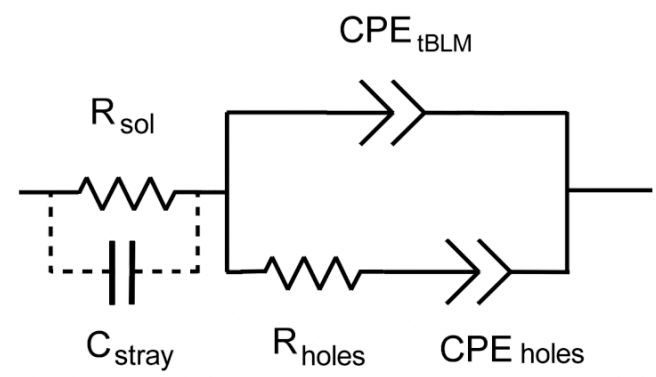

Table S2: Model parameters:"

\begin{tabular}{lccl}
\hline \multicolumn{1}{c}{ Parameter } & POPC tBLM & DPhyPC tBLM & \multicolumn{1}{c}{ Units } \\
\hline$C P E_{\mathrm{tBLM}}$ & $1.06(1)$ & $0.90(1)$ & $\mu \mathrm{F} \cdot \mathrm{cm}^{-2} \cdot \mathrm{s}^{(\alpha-1)}$ \\
$\alpha_{\mathrm{tBLM}}$ & $0.984(1)$ & $0.986(1)$ & \\
$C P E_{\text {holes }}$ & $4.10(6)$ & $1.88(4)$ & $\mu \mathrm{F} \cdot \mathrm{cm}^{-2} \cdot \mathrm{s}^{(\alpha-1)}$ \\
$\alpha_{\text {holes }}$ & $0.395(22)$ & $0.475(26)$ & \\
$R_{\text {holes }}$ & $52500(6400)$ & $89400(13100)$ & $\Omega \cdot \mathrm{cm}^{2}$ \\
\hline \hline$R_{\text {sol }}$ & $24.4(2)$ & $20.4(1)$ & $\Omega \cdot \mathrm{cm}^{2}$ \\
$C_{\text {stray }}$ & $0.014(1)$ & $0.020(2)$ & $\mu \mathrm{F} \cdot \mathrm{cm}^{-2}$ \\
$\chi^{2}$ (fit quality) & $5.68 \cdot 10^{-5}$ & $6.79 \cdot 10^{-5}$ & \\
\hline
\end{tabular}

"Shown is a representative set of fitting parameters obtained from a single EIS spectrum each of the tBLMs as indicated. Some of the model parameters, such as $C P E_{\text {holes, }}$ and $R_{\text {holes }}$, showed significant variability $(>25 \%)$ from sample to sample, while others, such as $C P E_{\mathrm{tBLM}}$, were reproducible within $10 \%$. The data are normalized to the geometric surface area, $a$. The surface roughness factor is $\beta \sim 1.49$. 